\title{
Erratum: Dynamics of Water Dissociative Chemisorption on Ni(111): Effects of Impact Sites and Incident Angles [Phys. Rev. Lett. 114, 166101 (2015)]
}

\author{
Bin Jiang and Hua Guo®
}

(Received 23 March 2020; accepted 25 March 2020; published 9 April 2020)

DOI: 10.1103/PhysRevLett.124.149901

In the Letter, we compared in Fig. S6 in the Supplemental Material the dissociation probabilities of $\mathrm{D}_{2} \mathrm{O}$ in various vibrational states on $\mathrm{Ni}(111)$ calculated by six-dimensional (6D) quantum dynamics (QD) and quasiclassical trajectory (QCT) methods with the 6D potential energy surface (PES) reported in our previous work [1] (Ref. 16 in the Letter). In Ref. [1], we constructed an original PES from density functional theory (DFT) calculations using the PW91 functional with a barrier height of $\sim 0.67 \mathrm{eV}$ and a revised PES with a barrier height increased by $\sim 0.10 \mathrm{eV}$ by a simple exponential adjusting function aimed at a better agreement with experiment. We now realize that those QD and QCT results in Fig. S6 were inadvertently taken from the original PW91 PES and the revised PES, respectively, making the comparison inappropriate. Here, we revise Fig. S6 by displaying the QCT reaction probabilities obtained using the same original PW91PES.

Figure S6 was used to show that both QD and QCT methods yield qualitatively similar trends with respect to the vibrational efficacy on the reactivity and the calculated dissociation probabilities are generally consistent. This statement remains true with the corrected Fig. S6, which does not alter any of our conclusions in the Letter. We emphasize that the correct QCT data based on the original 6D PW91 PES were used in Fig. 3 in that Letter. It is also worth noting that the correct 6D QCT reaction probabilities are actually higher than the QD counterparts. This result is reasonable and consistent with a more accurate comparison on the nine-dimensional PES in our recent work [2], likely caused by the spurious zero point energy leakage in the QCT calculations.

We thank Prof. Geert-Jan Kroes for bringing this issue to our attention.

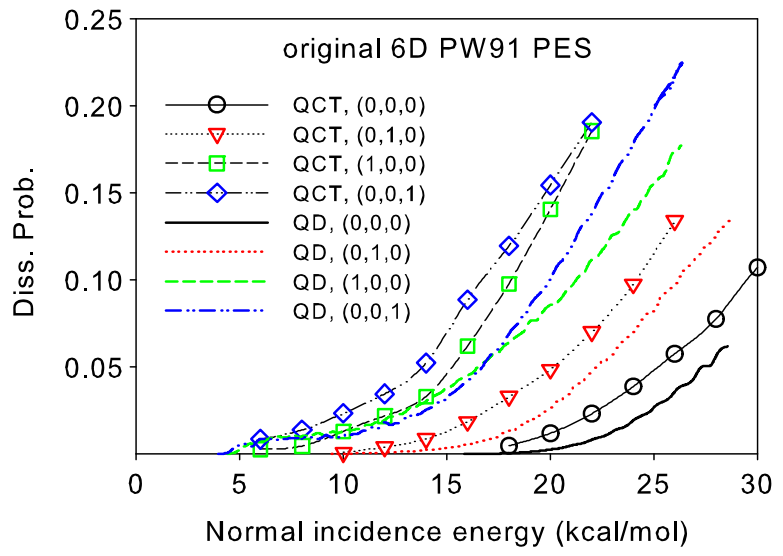

Fig. S6. Comparison of dissociation probabilities for $\mathrm{D}_{2} \mathrm{O}$ in its ground and vibrationally excited states obtained by quasiclassical trajectories (QCT) and quantum dynamics (QD) using the original 6D PW91 PES in Ref. [1]. More details can be found in the main text.

[1] P. M. Hundt, B. Jiang, M. van Reijzen, H. Guo, and R. D. Beck, Science 344, 504 (2014).

[2] Q. Liu, L. Zhang, Y. Li, and B. Jiang, J. Phys. Chem. Lett. 10, 7475 (2019). 